\title{
Traumatic Intradural Lumbar Disc Herniation without Bone Injury
}

\author{
Hyun-Woo Lee, Young-Min Kwon \\ Department of Neurosurgery, Dong-A University College of Medicine, Busan, Korea
}

Intradural lumbar disc herniation is a rare disease. According to the reports of intradural lumbar disc herniations, most cases have developed as a chronic degenerative disc diseases. Traumatic intradural lumbar disc herniations are even rarer. A 52-year-old man visited our emergency center with numbness in his left calf and ankle after falling accident. Initial impression by radiologic findings was a spinal subdural hematoma at the L1 level. A follow up image two weeks later, however, did not demonstrate any interval change. The patient was decided to have an operation. In operative findings, a ruptured disc particle penetrating the ventral and dorsal dura was indentified after laminectomy. It was assumed to be a traumatic outcome not a degenerative change.

Key Words: Intradural disc herniation · Subdural hematoma • Lumbar spine

\section{INTRODUCTION}

Intradural disc herniation (IDH) is a rare disease and its incidence is reported as $0.26-0.30 \%$ of all disc herniations ${ }^{16}$. Approximately $92 \%$ of all IDHs occur in the lower lumbar spine $^{5,11)}$. IDHs rarely occur in the upper lumbar spine ${ }^{11,13,15)}$. IDHs primarily occur as a result of chronic degenerative diseases and rarely occur due to traumatic events ${ }^{1,14}$. Traumatic lumbar spine lesions usually result in bony fractures and paraspinal soft tissue injuries rather than isolated disc herniations. In this report, we present a rare case of a patient who exhibited an intradural mass lesion at L1, which initially mimicked a spinal subdural hematoma (SDH) and was later determined to be an IDH without an accompanying lumbar spine bony fracture after a traumatic event.

\section{CASE REPORT}

A 52-year-old man presented at the hospital with numbness in his left calf and ankle after falling accident two days ago. The patient was initially admitted at another medical center after the accident but transferred to our hospital at his request.

- Received: May 31, 2013 - Revised: August 3, 2013

- Accepted: August 5, 2013

Corresponding Author: Young Min Kwon, MD

Department of Neurosurgery, College of Medicine, Dong-A University,

Dongdaesin-dong 3-ga, Seo-gu, Busan 602-715, Korea

Tel: +82-51-240-5241, Fax: +82-51-242-6714

E-mail:ymkwon@dau.ac.kr
A motor power grade was intact with slightly decreased sensation along the left $\mathrm{L} 5$ dermatome. The patient did not have back pain. Bladder and bowel functions were intact, and no other significant abnormalities were found upon neurological examination.

In his medical history, he had visited our hospital six weeks ago with complaints of radiating pain in his right leg along the $\mathrm{L} 5$ dermatome after falling down from $2 \mathrm{~m}$ height. At that time, his lumbar spine magnetic resonance image (MRI) scan exhibited diffuse bulging intervertebral disc in L4-5 (Fig. 1). The patient had received a conservative treatment with a non-steroidal anti-inflammatory drug and discharged with the improvement of symptoms.

At this time, the patient's plain radiography did not present bony abnormalities such as fractures or dislocations. In his lumbar computed tomography (CT) scan, a small calcified lesion was found at the L1-2 level, posterior to the disc (Fig. 2). On his lumbar T2-weighted MRI, a high signal intensity with
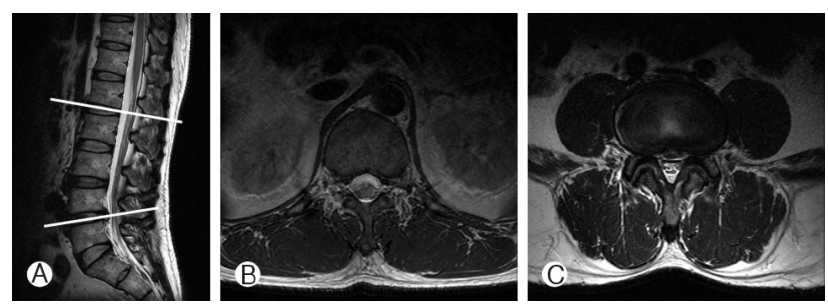

Fig. 1. Lumbar spine magnetic resonance images of six weeks ago. T2 sagittal image presents mild disc protusion in L4-5 disc level (A). Intradural mass or other abnormalities are not detected in L1-2 disc level (B). Disc protusion is seen on axial T2 image in L4-L5 disc level (C). 

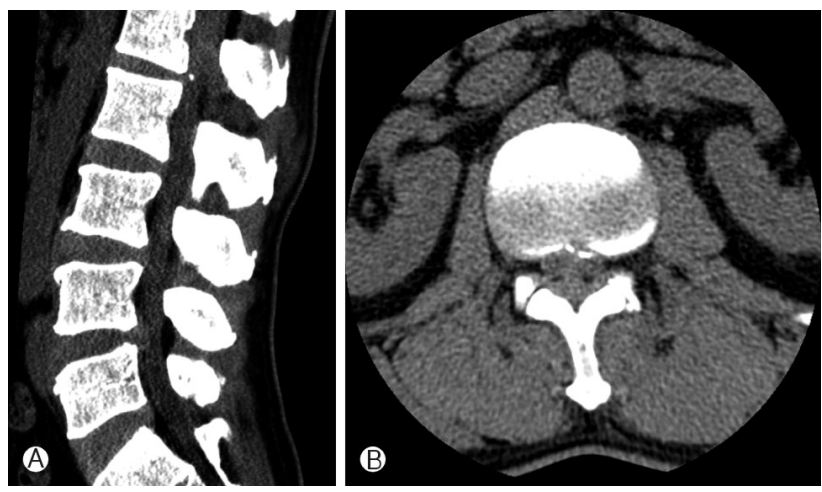

Fig. 2. Computed tomography $(C T)$ presents calcified lesion on posterior portion in L1-2 disc level on sagittal (A) and axial (B) view. Any bony abnormalities or epidural gas are not detected.
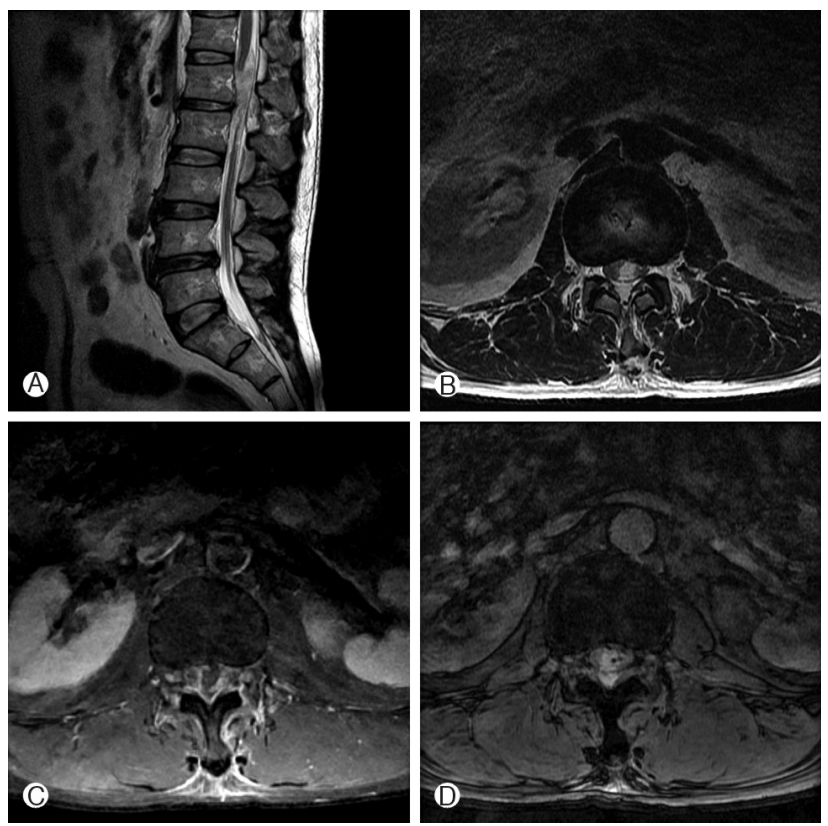

Fig. 3. Lumbar spinal MRI of the patient. A intradural mass with high signal intensity is demonstrated in T2 weighted sagittal (A) and axial (B) image. Intradural mass presents mild rim enhancement in gadolinium-enhanced $\mathrm{T} 1$ image $(\mathrm{C})$ and heterogeneous low signal intensity with signal void in gradient echo image (D). Compared with previous lumbar spinal MRI, the height of L1-2 disc is decreased and the signal intensity of it is also deminished.

an amorphous shape lesion was located intradurally and it compressed the spinal cord anteriorly at the L1 level (Fig. 3A, B). In his gadolinium-enhanced MRI, the lesion exhibited mild rim enhancement (Fig. 3C) and it presented heterogeneous low signal intensity in the gradient echo (GRE) image (Fig. 3D). We considered the lesion to be a spinal SDH, and decided to perform a conservative treatment. At the follow up MRI scan after two weeks of treatment, the lesion still remained unchanged without any improvement of numbness, so we deci-
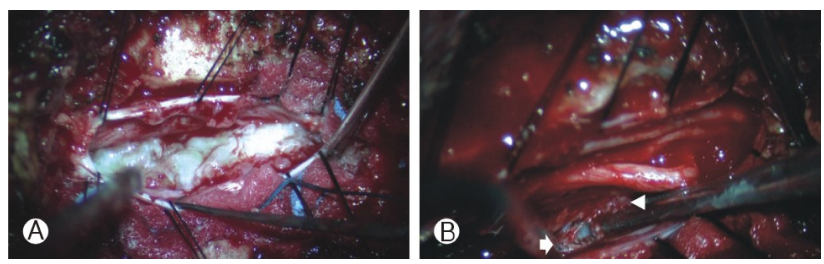

Fig. 4. Intraoperative findings after durotomy (A). Soft and whitish ruptured disc particle is identified among cauda equina. A hard bony spur (arrow head) was detected at the ventral side and a dural defect (arrow) was observed (B).

\section{ded to operate.}

A subtotal laminectomy was performed at L1, which revealed an out-punching whitish soft particle on the dorsal dura. The particle was thought to be a ruptured disc material and removed gently, then sent for pathology. The pathologic results determined the particle to be degenerating fibrocartilagenous tissue corresponding to an intervertebral disc. After removing the extradural lesion, a midline durotomy was done from the dura defect uncovering large, cartilaginous disc particles in the middle of the intradural space (Fig. 4A). After removing the disc particles, a hard bony lesion remained adhesively to the ventral dura and a small ventral dura defect was identified around the bony lesion (Fig. 4B). The hard bony lesion was thought to be a calcified lesion at the posterior L1/2 intervertebral disc in his lumbar spinal CT scan. We tried to repair the dura defect to protect a leakage of cerebrospinal fluid (CSF), but the ventral dura tear could not be repaired due to its severe adhesion. A watertight closure on dorsal dura was performed. After the operation, the patient's numbness in his left leg has gradually improved. Two weeks after surgery, the patient was discharged from the hospital with free of symptoms.

\section{DISCUSSION}

IDHs are rare clinical diagnoses that typically occur in patients aged in their fifties ${ }^{11)}$. Dandy ${ }^{4}$ first reported an intradural lumbar disc herniation in 1942 and the incidence of IDHs is reported between 0.04 and $0.33 \%$ of all reported lumbar disc herniations" ${ }^{9}$. The most common site of intradural lumbar disc herniations is the L4-5 (55\%) area, followed by L3-4 (16\%), and L5-S1 (10\%), far fewer occur at L1-2 and L2-3 $3^{11,13,15)}$. Furthermore, traumatic IDHs are rarer events and the incidence rate has not yet been reported in the literature. Most traumatic disc herniations in the lumbar spine frequently have severe bony injuries such as fractures and dislocations ${ }^{8)}$. In our case, however, we found an IDH after a traumatic event without fractures or dislocations. 
Although the mechanisms that cause disc herniations to pass the dura mater are unclear, the widely accepted hypothesis states that adhesion between the ventral dura and posteriorlongitudinal ligament (PLL) leads to the successive perforation of these firmly adhesive tissues, including the annulus fibrosus, due to the increased intradiscal pressure ${ }^{1,4,9,10)}$. Dandy ${ }^{4)}$ explained that sudden pressure on the protruded disc might erode and then penetrate the overlying ventral dura. Other reports have suggested that dense adhesion, whether it is congenital or acquired, fixate the dural $\mathrm{sac}^{1,14)}$.

Diagnosing an IDH is difficult due to its rarity. Diagnosis using radiological images is a difficult task, because lumbar IDHs can have various radiological and clinical features ${ }^{3,17}$. Furthermore, lesions may be ignored in simple radiography and CT scans, which are one of the most frequently used radiologic examinations in the diagnosis of spinal injuries. Several radiologic findings that suggest the presence of IDHs have been reported. Hidalgo-Ovejero et $\mathrm{al}^{6}{ }^{6}$ reported that the presence of epidural gas in a CT scan could be a clue of the presence of an IDH. In our case, however, we could not find epidural gas on the CT scan. Since Wasserstrom et al. ${ }^{18)}$ made the first IDH diagnosis using a gadolinium-enhanced MRI in 1993, the rim enhancement of herniated discs has been accepted as a typical MRI finding in IDHs. Furthermore, Choi et $\mathrm{al}^{2)}$ presented the loss of PLL continuity and a sharp beaklike feature on a T2-weighted image as indicators of IDH. In our case, we also observed a peripheral rim enhancement pattern on the gadolinium-enhance T1 MRI, but it was not enough to consider the IDHs due to the small size of portion (Fig. 3C). Moreover, the loss of PLL continuity was not definite on our initial lumbar spine MRI. Therefore, our initial impression of the intradural mass at L1 was a spinal SDH, although a traumatic spinal SDH is also very rare ${ }^{12)}$. On MRI, the spinal SDH yielded high signals on both T1 and T2, but the signal intensity of the spinal SDH on the MRI also depend on the duration ${ }^{7}$. In our case, the intradural lesion exhibited high signal-intensity on the T2 scan (Fig. 3A, B) and an isosignal on the T1 scan. On the GRE scan, however, the intradural lesion had a high signal-intensity with a low signal void inside the intradural lesion (Fig. 3D), thus we assumed it was a spinal SDH. An intradural abscess could be considered as another differential diagnosis, but we excluded it because there were no signs of infection such as fever or leukocytosis.

Additionally, the patient had visited our hospital six weeks previously for radiating pain in his right lower leg. At that time, the patient checked a lumbar MRI scan, and was diagnosed with a diffuse bulging disc in L4-5 and there was no intradural mass lesion. Compared with the MRI from that visit, de-novo intradural lesion was detected. Moreover, the height and the signal intensity of the intervertebral disc of L1-2 had decreased in the recent study (Figs. 1A and 3A). We assumed that a strong vertical pressure had been applied to cause the intervertebral disc rupture, then the ruptured particles penetrated the PLL, ventral dura, and through dorsal dura to the epidural space. According to the clues mentioned above, we could assume that the IDH developed from a trauma rather than a degenerative condition.

\section{CONCLUSION}

We experienced a rare case of a lumbar IDH after trauma without an accompanying bony fracture or dislocation. For patients with an intradural lesion at the lumbar spine after trauma, there may be various diseases that can be differentiated. As with the patient in this report, IDHs should be considered as differential diagnoses and prompt removal of the intradural mass is necessary.

\section{REFERENCES}

1. Blikra G: Intradural herniated lumbar disc. J Neurosurg 31: 676-679, 1969

2. Choi JY, Lee WS, Sung KH: Intradural lumbar disc herniationIs it predictable preoperatively? A report of two cases. Spine 7:111-117, 2007

3. D’Andrea G, Trillò G, Roperto R, Celli P, Orlando ER, Ferrante $\mathrm{L}$ : Intradural lumbar disc herniations: the role of MRI in preoperative diagnosis and review of the literature. Neurosurg Rev 27:75-80; discussion 81-82, 2004

4. Dandy WE: Serious complications of ruptured intervertebral disks, JAMA 119:474-477, 1942

5. Han IH, Kim KS, Jin BH: Intradural Lumbar Disc Herniations Associated with Epidural Adhesion: Report of Two Cases. J Korean Neurosurg Soc 46:168-171, 2009

6. Hidalgo-Ovejero AM, García-Mata S, Gozzi-Vallejo S, IzcoCabezón T, Martínez-Morentín J, Martínez-Grande M: Intradural disc herniation and epidural gas: something more than a casual association? Spine 29:E463-E467, 2004

7. Hung KS, Lui CC, Wand CH, Wang CJ, Howng SL: Traumatic Spinal Subdural Hematoma with Spontaneous Resolution. Spine 27:E534-538, 2002

8. Jang JW, Lee JK, Seo BR, Kim SH: Traumatic Lumbar Intradural Disc Rupture Associated With an Adjacent Spinal Compression Fracture: Spine 35:E726-729, 2010

9. Kataoka O, Nishibayashi Y, Sho T: Intradural lumbar disc herniation. Report of three cases with a review of the literature. Spine 14:529-533, 1989

10. Kim HS, Eun HP, Park JS: Intradural Migration of a Sequestrated Lumbar Disc Fragment Masquerading as a Spinal Intradural Tumor. J Korean Neurosurg Soc 52:156-158, 2012

11. Koç RK, Akdemir H, Oktem IS, Menkü A: Intradural lumbar disc herniation: report of two cases. Neurosurg Rev 24:44-47, 2001 
12. Lyo IU, Kwon SC, Sim HB: Traumatic Acute Spinal Subdural Hematoma - A case report - Kor J Spine 2(4):369-372, 2005

13. Mailleux R, Redant C, Milbouw G: MR diagnosis of transdural disc herniation causing cauda equine syndrome. JBR-BTR 89 : 303-305, 2006

14. Mut M, Berker M, Palaoğlu S: Intraradicular disc herniations in the lumbar spine and a new classification of intradural disc herniations. Spinal Cord 39:545-548, 2001

15. Paul MA, Yosafe TW: Intradural disc herniation at L1-L2: report of two cases. J Spinal Cord Med 34:312-4, 2011
16. Pradeep KS, Sandeep S, Rahesh D, Pankaj B, Sharad G: Dorsal Herniation of Cauda Equina Due to Sequestrated Intradural Disc: Asian Spine Journal 6, 145-147, 2012

17. Tatsuro S, Kiyoshi H, Noboru Y, Kazuo I: The Specific Sagittal Magnetic Resonance Imaging of Intradural Extra-Arachnoid Lumbar Disc Herniation. Case Rep Med 383-451, 2012

18. Wasserstrom R, Mamourian AC, Black JF, Lehman RA: Intradural lumbar disk fragment with ring enhancement on MR. AJNR Am J Neuroradiol 14:401-404, 1993 